\title{
Análisis de los discursos de los y las jóvenes sobre la violencia en las relaciones de pareja
}

\author{
Analysis of the speeches of the young people on violence in intimate relationships
}

\author{
Yolanda Rodríguez Castro*, Patricia Alonso Ruido* \\ *Facultad de Ciencias de la Educación, Universidad de Vigo (España)
}

\begin{abstract}
Resumen
El objetivo de nuestro estudio consiste en analizar la violencia en las relaciones de pareja de los/as adolescentes a través de sus propios discursos a través de grupos de discusión. Para ello, se llevó a cabo un total de cuatro grupos de discusión, dos en el $2^{\circ}$ ciclo de la ESO y otros 2 en Bachillerato en un Instituto de Educación Secundaria de la ciudad de Ourense. En total participaron 40 estudiantes con edades comprendidas entre 15 y 18 años. Los resultados evidencian que chicos y chicas muestran percepciones de las relaciones de pareja; las chicas siguen mostrando en sus discursos visiones más románticas del amor, y siguen considerando los celos como una muestra de amor. En cuanto a la violencia ejercida en las parejas de adolescentes, son muchas chicas las que afirman conocer distintos casos de amigas que sufren violencia física leve y violencia psicológica/verbal. También los chicos manifiestan conocer casos de violencia verbal por parte de sus parejas femeninas. La violencia de género está inmersa en su entorno.

Palabras clave: violencia de género, adolescentes, pareja.
\end{abstract}

\section{Abstract}

The aim of our study was to analyze violence in relationships of the teenagers through their own speeches through discussion groups. For this, a total of four focus groups, two in the second cycle of E.S.O. and other two in Bachillerato was held in the Secondary School of the city of Ourense. In total 40 students participated aged between 15 and 18 years. The results show that boys and girls show perceptions of relationships; girls continue to show in his speeches more romantic visions of love, jealousy and still considered as a token of love. As for violence in adolescent couples, many girls who claim to know many cases of women who have mild physical violence and psychological/verbal violence. Also the guys know manifest cases of verbal violence by their female partners. Gender violence is embedded in its environment.

Keywords: domestic violence, teenage, couple.

La violencia en las relaciones de pareja entre adultos en España y en particular en la población adolescente y joven, se está convirtiendo en uno de los problemas sociales más graves.

La violencia en las relaciones de pareja entre jóvenes es conocida en la litereratura internacional con el término Dating Violence que se define como "la amaneza o el uso del abuso físico, sexual o verbal por uno de los miembros de la pareja sobre el otro" (Anderson \& Danis, 2007, p. 88). Lo que implica reconocer que para identificar adecuadamente las situaciones de Dating Violence es insuficiente evaluar las agresiones físicas y/o sexuales, sino que hay que tener en cuenta otras formas de violencia como las agresiones verbales y las amenazas, que a menudo ocurren de forma simultánea o que preceden a las agresiones físicas o sexuales (Capaldi \& Crosby, 1997). La gravedad de esta problemática se intensifica ya que esta violencia en las parejas más jóvenes se produce cada vez a una edad más temprana (Kury, Obergfell-Fuchs \& Woessner, 2004; Price \& Byers, 1999). Esta violencia en las relaciones de pareja afecta tanto a las parejas que llevan juntas mucho tiempo como las que están empezando una relación de noviazgo o incluso la violencia que se produce en las primeras citas (Centers for Disease Control and Prevention, 2012).

Son pocos los estudios realizados en España sobre Dating violence, estos son realizados mayoritariamente en la última década. Uno de los primeros es el de González y Santana (2001) que informa que el 7.5\% de los chicos y el $7.1 \%$ de las chicas reconocen que en una o más ocasiones han pegado o empujado a su pareja; mientras que un $23.9 \%$ de los chicos y un $28.8 \%$ de las chicas manifiestan utilizar en alguna ocasión violencia verbal. Más recientemente, Muñoz-Rivas, Rodríguez, Graña, O'Leary y González (2007) exponen que entre el $60-70 \%$ de los estudiantes encuestados manifiestan que en algún momento habían agredido verbalmente a su pareja, mientras que el $14 \%$ reconoce haber ejercido violencia física. Las formas más severas de violencia física afectan al $2.9 \%$ de los chicos y al $1.3 \%$ de las chicas.

En esta dirección, se manifiesta la necesidad de analizar la relación entre el amor y la violencia de género en los/as adolescentes. Por tanto, el objetivo de nuestro estudio consiste en analizar la violencia en las relaciones de pareja de los/as adolescentes a través de sus propios discursos a través de grupos de discusión.

\footnotetext{
Método

Participantes

Los/as participantes del presente estudio fueron alumnos/as matriculados/as en el segundo Ciclo de Educación Secundaria Obligatoria y Bachillerato., de un Instituto de educación Secundaria de la ciudad de Ourense.

La muestra quedó formada por 34 estudiantes con edades comprendidas entre 15 y 18 años, de los cuales 19 son chicos y 15 son chicas.
} 


\section{Instrumentos}

Se utilizó como instrumento de investigación los grupos de discusión o "conversación etnográfica" (Renold, 2005). De esta forma a través de un guion de preguntas de carácter semi-estructurado se les plantearon cuestiones relativas a qué significa una relación de pareja, que entendían por violencia en las relaciones de pareja o noviazgo entre adolescentes o si conocían algún amigo/a que sufriera este tipo de violencia de pareja. Por último, se les preguntó también sobre cuales creían que eran las razones por las que se puede actuar con violencia hacia la parece.

\section{Procedimiento}

Inicialmente, se llevó a cabo la toma de contacto con el centro en el que se iba a llegar a cabo la investigación. Dicho centro fue informado del procedimiento y finalidad de la investigación. Se contó con el consentimiento de padres y madres como requisito para la participación del alumnado en la presente investigación.

Las sesiones fueron grabadas a través de dispositivos de recogida de voz por un investigador. Se desarrollaron en el propio centro académico, con una duración de 40-50 minutos, dentro del horario lectivo.

Por motivos de confidencialidad y rigor científico para preservar la identidad de cada participante, se asignó a cada uno/a un código que significa GD (grupo de discusión), 1, 2, 3 o 4 (el número del grupo correspondiente), chico/chica (género de cada alumno o alumna), seguido del número del o de la alumna que verbaliza dicho comentario y finalmente 1. (línea) relativo a la línea en la que se encuentra cada expresión en la transcripción literal.

\section{Resultados}

Los resultados evidencian que chicos y chicas muestran percepciones similares de las relaciones de pareja. Por un lado en lo referente a que entienden por una relación de pareja, reflejan expresiones como, que se quieren, se respetan y que son fieles:

"Se quieren, se dan cariño, tienen confianza..." (GD1, chica2, 1. 12).

"Ser fieles, se aceptan, sinceridad" (GD2, chico2, 1. 22).

"Con la que puedes contar más que con las demás y con la que sientes algo especial" (GD4, chica2, 1. 956-957).

"Una persona con la que tienes muchos lazos, con la que puedes compartir experiencias que no compartes con otra gente, como relaciones sexuales" (GD4, chica7, 1.954-955).

"Puede ser una persona en la que confías más" (GD4, chica2, 1.955-956).

Por otro lado en sus discursos suryacen tambien expresiones en torno a los mitos del amor romántico, tales como:

"Yo creo que es bastante complicado encontrar a esa persona ideal, que si la encuentras es una suerte, pero que no es nada fácil" (GD4, chico3, 1.988-989).

"Bueno que haya una persona que sea tu media naranja sí, pero la necesidad de encontrarla..." (GD3, chica6, 1.438-439).

"No sé, si te enamoras es porque es para siempre, ¿no?” ( GD3, chica6, 1.440-443).

No obstante son las chicas las que siguen mostrando en sus discursos visiones más románticas del amor en torno, como:

"Es darlo todo por esa persona. Entregar el $100 \%$ por una persona" (GD1,chica6, 1.20-21).

"Hacer cosas que no harías por otra persona" (GD1_chica2, 1.21).

"No puedes dejar de pensar en ella o en él " (GD4, chica 7, L.964).

"No sé, es difícil definirlo, o sea, es algo que se siente pero no puedes explicar lo que es un cúmulo de sentimientos que tan pronto, si eres correspondido está todo muy bien, y si no lo eres te hundes en la mierda" (GD2, chica3, 1.1337-1339).

"Tranquilidad" (GD3, chica5, 1.415).

"Sí [el amor lo puede todo] tu ya puedes tener 25 exámenes que esa persona te dice un nada y ya está, te despreocupas por todo" (GD4, chica1, 1.112-113).

Por otro lado, en su discurso se evidencia que siguen considerando los celos como una muestra de amor, explicando que:

"Pues, los celos están bien, si la quieres algo, te tienes que celar" (GD1_chico9, 1.101).

"Sí, es preocuparte por la otra persona" (GD1_chica5, 1.91).

"Hay celos que son obsesivos y no son buenos, que acaban por agobiarte, pero tú siempre vas a estar celosa de alguien es porque sientes algo por esa persona" (GD1, chica1, 1.95-97).

"Claro, que te importa" (GD3, chico 2, 1.454).

"Si tu estas celoso de una persona, es porque la quieres, porque quieres estar con ella y no con otra" (GD2, chico1, 1.1393-1394).

"Si no sientes celos es...como si no tienes sangre" (GD4, chico1, 1.998-999).

“A ver yo creo que si tienes algo de celos es bueno, porque manifiestas que tienes cariño a esa persona o algo que te importa" (GD4, chica2 1.1001-1002).

No obstante en algunos casos sí manifiestan cierto rechazo a los celos en sus relaciones de pareja, expresando que:

"Hay celos sanos, celos hasta cierto punto, después..." (GD2, chico7, 1.1397-1398).

"Hay celos que matan" (GD2, chico9, 1.1395-1396).

En referencia a la conceptualización que crean de partida que tenían sobre lo que entienden por violencia en las relaciones de pareja adolescentes, destacan expresiones como:

"Psicológica y física, cualquiera." (GD2, chica3, 1.1435)

"Ya no sólo eso, sino intentar que la otra persona se sienta mal para que cambies [...] con el típico si me quisieras..." (GD2, chico4, 1.1437-1439).

"Sometimiento" (GD1, chica3, 1.151).

"Humillación, falta de respeto" (GD1, chica2, 1.152).

"En la pareja yo creo que es más...un sentimiento de posesión hacia la otra persona" (GD4, chico4, 1.1030-1031). 
"Creerte que siempre tienes la razón" (GD3, chico2, 1.494).

"Tratar a tu pareja inferiormente a ti, hacerle daño" (GD3, chico3, 1.493-494).

En cuanto a la violencia ejercida en las parejas de adolescentes, son muchas chicas, y algún chico, las que afirman conocer distintos casos de amigas que sufren violencia física leve y violencia psicológica/verbal. Enumeran situaciones como:

“Ah, sí, una chica del instituto, hace unos años o así tuvo un novio que la maltrataba, empezó como a hacerle así en la cabeza, y después la agarraba fuerte del brazo, no le dejaba ir con sus amigas y todo eso. Hasta le llegó a pegar a su madre... según ella me contó y buff...con 14 años." (GD3, chica5, 1.505-508).

"Sí, yo conozco a una chica, que creo que de aquí la conoce más gente, que el novio la maltrataba física y verbalmente y tuvieron que recurrir hasta una orden de alejamiento y el chico la seguía acosando y eso." (GD1, chico6, 1.1079-1081).

"Yo sé de una que sucedió hace una semana, de un chico que estaba fueron dos líos con esa chica y él pasó de ella y ella es un poco choni, choni perdida y le fue allí a insultarle y a pegarle, y claro, mi amigo es moro y coge ella y le empieza a pegar, el estaba pasando, le iba a tirar el cigarro a la chica y le iba a apagar el cigarro en la cara, en el ojo y cogió él y le rompió los dientes de un puñetazo. Yo no lo vi, pero he visto a la chica y me lo han contado todos los que estaban allí." (GD2, chica3, 1.1464-1469).

También los chicos manifiestan conocer casos de violencia verbal por parte de sus parejas femeninas: "Demasiada, en cuanto hay una discusión" (GD2, chico4, 1.1440-1441).

En cuanto a las razones que señalan para que algunos/as actúen con violencia hacia sus parejas, son principalmente los celos y el consumo excesivo de alcohol u otras drogas, señalando que:

"Si, yo sí. A ver, porque el chico bebía, y en plan una noche la chica fue a junto el novio y si que le pegó. Y ahora ya no están juntos, ero la primera vez ella le perdonó. Pero yo conozco a más de una chica a la que le pegaron. Yo conozco a otra chica que el novio también estaba borracho y le pegó. Pero la primera sí que lo vimos las demás, pero la segunda no nos lo contó hasta después, se lo calló." (GD4, chica7, 1.1074-1078).

"Por cualquier motivos, por celos o no sé por desconfianza o cualquier cosa que la trates de una manera que no se merece, no sé, algún motivo siempre hay ¿no? o no..." (GD1, chica5, 1.153-155).

"Por celos muchas veces, por cualquier cosa que te moleste es muy impulsivo" (GD4, chica4, 1. 1321-1322).

"cuando estas drogado no controlas lo que haces..., le das una bofetada $y$ el día siguiente no te acuerdas..."(GD1, chica4, 1. 302-303).

Destacar que un par de chicas manifestaron que cuando una chica "insulta" a su pareja es para defenderse de los ataques de su pareja, expresando que: "Es que a veces para defenderte lo insultas o así, aunque te dan ganas de darle una bofetada" (GD1, chica5, 1. 282-283).

\section{Discusión}

En referencia al análisis sobre violencia en las relaciones de pareja adolescentes hemos encontrado que tanto los chicos como las chicas muestran percepciones similares a la hora de definir una relación de pareja. Estos resultados están en la línea de los obtenidos en el estudio de Amurrio, Larrinaga, Usategui y del Valle (2008) que comprueban que las relaciones se caracterizan por la confianza, sinceridad y respeto, es decir, una serie de principios éticos que garantizan la igualdad en la pareja.

En relación a las visiones del amor los chicos y las chicas muestran posiciones desiguales. Encontramos evidencias en otras investigaciones que apoyan nuestros resultados. El estudio realizado por Barrón, Martínez-Iñigo, De Paul, \& Yela (1999) donde la mayoría las personas entrevistadas muestran altos niveles de aceptación de los mitos como el de la media naranja. No obstante son las chicas las que en mayor medida manifiestan la idealización del amor romántico, resultados en la línea a los obtenidos por otros estudios anteriores como el de Ferrer, Bosch, Navarro, Ramis y García (2008) donde se afirma que entre los hombres predomina el amor sexual y entre las mujeres el romántico. Otras investigaciones sobre los mitos del amor romántico apoyan nuestros evidencias, como por ejemplo el estudio de Ferrer, Bosch y Navarro (2010) donde encontraron que la mayoría de los/as participantes creían en el mito de la omnipotencia o la media naranja.

En relación a los celos, nuestros resultados demuestran que siguen siendo considerados por los y las adolescentes una muestra de amor. De la Peña, Ramos, Luzón y Recio (2011) también observan que la interiorización de la creencia de que los celos son una muestra de amor, incluso un requisito indispensable de un verdadero amor, es muy elevada entre la juventud. Esta tendencia es preocupante puesto que dicha falacia sobre los celos suele usarse habitualmente para justificar en las parejas comportamientos egoístas, injustos, represivos $\mathrm{y}$, en ocasiones, violentos.

En lo relativo a la violencia detectada en la presente investigación, hemos encontrado que de todos/as los/as participantes nadie admitió haber sufrido o perpetrado actos de violencia física. Supone un resultado diferente al de otros estudios como el de Krahé y Berger (2005), que concluía que un $44 \%$ de los jóvenes habían admitido ser víctimas de agresiones físicas leves alguna vez. En esta línea otras investigaciones revelaban que un $22.8 \%$ de los y las adolescentes que estaban implicados en agresión física a sus parejas, mientras que un $29.6 \%$ lo estaban en victimización (Viejo, 2012).

Nuestros resultados revelan que los y las adolescentes consideran más frecuente violencia psicológica/verbal que la violencia física leve. Por el contrario, Samaniego y Freixas (2010) en su estudio con alumnos de la E.S.O., detectaron que el tipo de violencia más frecuentemente identificado es la violencia física directa. Otros resultados apoyan esta afirmación afirmando que se debe a que la sensibilidad hacia el maltrato y su percepción ganan en intensidad y nitidez en situaciones de violencia 
o acoso explícito (Amurrio, Larrinaga, Usategui, \& del Valle, 2008).

Generalmente tanto los chicos como las chicas atribuyen las agresiones físicas al consumo de alcohol o drogas. Muñoz, Gámez, Graña y Fernández (2012) confirman también que a mayor consumo, se observa una mayor violencia. Esta relación se fundamentaría en el hecho de que el consumo de alcohol y otras sustancias altera la percepción de los patrones de interacción y comunicacionales en la pareja, por lo que es más probable que surjan comportamientos violentos. Otras aportaciones (Guzmán et al., 2009) mantienen que el consumo de sustancias altera el juicio de realidad, impidiendo reconocer situaciones de peligro y disminuyendo las conductas de autoprotección, por lo que facilitaría la probabilidad de ser víctima de violencia, especialmente física y sexual.

Finalmente observamos que tanto que tanto chicos como chicas se ven envueltos en las dinámicas de las relaciones violentas, generándose así un doble rol víctima y agresor. Algunas investigaciones afirman que este hecho se debe a la sobrestimación de la violencia sufrida por los chicos y a la subestimación de la misma sufrida por las chicas (Bowker, 1998; Kimmel, 2006; Rodríguez, Lameiras, Carrera, \& Vallejo, 2013). No obstante, destaca especialmente el hecho de que las chicas puedan utilizar la violencia como acto de defensa en las relaciones de pareja violentas en las que se encuentran.

Por lo tanto, a la vista de los resultados obtenidos se evidencia que la violencia de género también está presente en la vida de los/as adolescentes, con lo cual es importante trabajar con este colectivo desde una perspectiva coeducativa que fomente el buen trato y una educación sin violencia.

\section{Referencias}

Amurrio, M., Larrinaga, A., Usategui, E., \& del Valle, A. I. (2008). Violencia de género en las relaciones de pareja entre adolescentes y jóvenes de Bilbao. Universidad del País Vasco. Facultad de ciencias sociales y de la comunicación, Departamento de Sociología, País Vasco.

Anderson, K. M., \& Danis, F. S. (2007). Collegiate sororities and dating violence: An exploratory study of informal and formal helping strategies. Violence Against Women, 13, 87-100.

Barrón, A., Martínez-Iñigo, D., De Paul, P., \& Yela, C. (1999). Romantic beliefs and myths in Spain. The Spanish Journal of Psychology, 2(1), 64-73.

Bowker, L. (1998). Masculinities and Violence. Thousand Oaks, California: Sage.

Callejo, J. (2002). Observación, entrevista y grupo de discusión: el silencio de tres prácticas de investigación. Revista Española de Salud Pública, 76 (5), 409-422.

Capaldi, D. M \& Crosby L. (1997). Observed and reported psychological and physical aggression in young, at-risk couples. Soc Dev, 6, 184-206.

Centers for Discase Control and Prevention. (2012). Understanding Teen Dating Violence. Recuperado de http://www.cdc.gov/ViolencePrevention/pdf/TeenDati
ngViolence2012-a.pdf

De la Peña, E. M., Ramos E., Luzón J. M. \& Recio P. (2011). Sexismo y Violencia de Género en la Juventud Andaluza. Instituto Andaluz de la Mujer, Consejería para la Igualdad y Bienestar Social, Junta de Andalucía

Ferrer, V. A, Bosch, E. \& Navarro, C. (2010). Los mitos románticos en España. Boletín de Psicología, 99, 7-31.

Ferrer, V. A, Bosch, E., Navarro, C., Ramis, M. C. \& García, E. (2008). El concepto de amor en España. Psicothema, 20 (4), 589-595.

González, R. \& Santana, J. D. (2001). La violencia en parejas jóvenes. Psicothema, 13, 127-131.

Gutiérrez, J. (2011). Grupo de Discusión: ¿Prolongación, variación o ruptura con el focusgroup? Cinta moebio, 41, 105-122. Disponible en www.moebio.uchile.cl/41/ gutierrez.html

Guzmán, F., Esparza, S., Alcántara, S., Escobedo, I., \& Henggeler, T. (2009). Consumo de alcohol en jóvenes y su relación con la violencia psicológica en el noviazgo. Revista electrónica Saúde Mental, Alcool e Droga, 5 (2), 3.

Kimmel, M. S. (2006) Manhood in America: A cultural history. New York: Oxford University Press.

Krahé, B. \& Berger, A. (2005). Sex differences in relationship aggression among young adults in Germany. Sex Roles, 52, 829-839.

Kury, H., Obergfell-Fuchs, J., \& Woessner, G. (2004). The extent of family violence in Europe. A comparaison of National Surveys. Violence Agaisnt Women, 10 (7), 749-769.

Muñoz, M. J., Gámez, M., Graña, J. L., \& Fernández, L. (2010). Violencia en el noviazgo y consumo de alcohol y drogas ilegales entre adolescentes y jóvenes españoles. Adicciones, 22, 125-134.

Muñoz, M. J., Graña J. L., O’Leary K. D., \& González M. P. (2007a): Aggression in adolescent dating relationships: prevalence, justification and health consequences. Journal of Adolescent Health, 40, 298 304.

Muñoz, M. J., Graña, J. L., O’Leary, K. D., \& González, M. P. (2007b): Physical and psychological aggression in dating relationships in Spanish university students. Psicothema, 19 (1), 102-107.

Price, E. L. \& Byers, E.S. (1999). The attitudes towards dating violence scales: Development and initial validation. Journal of Family Violence, 14, 351-375.

Renold, E. (2005). Girls, Boys and Junior Sexualities: Exploring Children's Gender and Sexual Relations in the Primary school. Londres: Routledge Falmer.

Rodríguez Castro, Y., Lameiras, M., Carrera, M. V., \& Vallejo, P. (2013) La violencia en las relaciones de pareja de los y las adolescentes: prevalencia y su relación con el sexismo y el amor. Summa Psicológica, 8(2), 1-22.

Samaniego, E. y Freixas, A. (2010). Estudio sobre la identificación y vivencia de violencia en parejas adolescentes. Apuntes de Psicología, 28 (3), 349-366.

Viejo, C. (2012). Dating violence y cortejo adolescente. Un estudio sobre la violencia en las parejas sentimentales de los jóvenes andaluces. Tesis doctoral. Universidad de Córdoba. 